\title{
Frequency Dependence of Output Voltage Generated from Bundled Compound Magnetic Wires
}

\author{
Yasushi Takemura, Member, IEEE and Akira Matsushita, Member, IEEE
}

\begin{abstract}
The output voltage generated from bundles of twisted Vicalloy wires was studied. When the magnetically soft layers of the wires were reversed by an applied external magnetic field, a pulse voltage, owing to a large Barkhausen jump, was induced in a search coil wound around the wires. The output did not depend on the exciting frequency of the applied field for the bundled wires with $n$ (number of bundled wires) $<5$. The frequency dependence for $n=10$ was attributed to the demagnetizing field in the thicker shape of the bundle. The bundled wires of $n=25$ exhibited dispersed peaks in output waveform due to a self-biasing effect, which was advantageous for obtaining a rectangular output waveform.
\end{abstract}

Index Terms-Magnetic sensor, power generation, Vicalloy wire.

\section{INTRODUCTION}

A TWISTED semi-hard magnetic wire of Vicalloy $(40 \mathrm{Fe}-50 \mathrm{Co}-10 \mathrm{~V})$ exhibits its coercive force of about 20 Oe in the outer shell and 60 Oe in the inner core [1]. When the magnetization of the soft layer is reversed parallel to that of the hard core by applying an external magnetic field $H_{e x t}$, a pulse voltage, owing to a large Barkhausen jump, is induced in a search coil wound around the wire. The amplitude of the voltage does not depend on $d H_{e x t} / d t$. This noble feature enables applications like rotation sensor, flow meter and others to detect "slow movements" as well as the pulse generating elements and electromagnetic transducers.

We have proposed a new method of power generation using a bundle of wires and indicated the advantages arisen from a demagnetizing field in the bundled wires [2]. In this paper, the frequency dependence of the output voltage generated from the bundled compound wires is discussed.

\section{EXPERIMENTS}

The magnetic wire used in this study was a semi-hard magnetic Vicalloy (40Fe-50Co-10V, wt $\%$ ) wire of $0.25 \mathrm{~mm}$ in diameter. The double layered magnetic structure was achieved by twisting the wire. This compound wire reveals a uniaxial magnetic anisotropy along the wire direction, and its coercive force is about $20 \mathrm{Oe}$ in the outer shell (soft layer) and $60 \mathrm{Oe}$ in the inner core (hard core) [1]. This element is essentially the same as the so-called Wiegand wire [3]. Fig. 1(b) shows the B-H curve of the compound magnetic wire. When the magnetization

Manuscript received October 10, 2000.

Y. Takemura is with Yokohama National University, Yokohama 240-8501, Japan (e-mail: takemura@dnj.ynu.ac.jp).

A. Matsushita was with Kanagawa University, Yokohama 221-8686, Japan. He is now with Nihon System Research Institute Inc., Kawasaki, Japan (e-mail: matusita@jasmine.ocn.ne.jp).

Publisher Item Identifier S 0018-9464(01)07097-2.

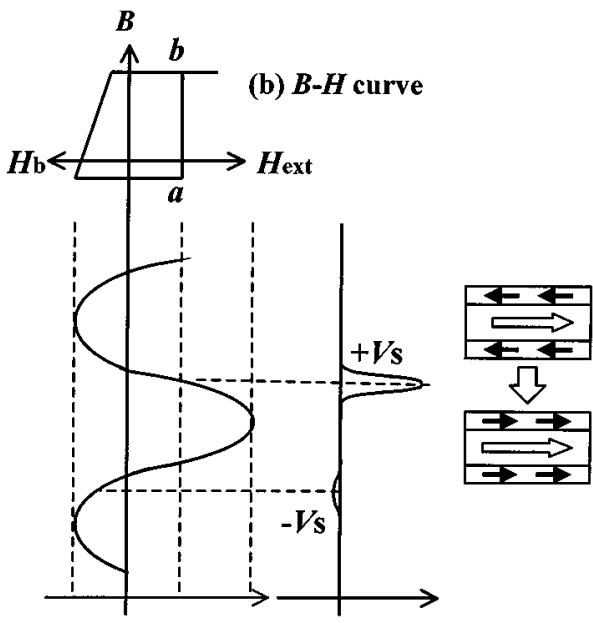

(a) Magnetic field (c) Output voltage

Fig. 1. Waveform of the external magnetic field (a), B-H curve of compound magnetic wire (b), output pulse voltage (c).

of the soft layer is reversed from antiparallel to parallel to that of the hard core (parallel magnetization corresponding to " $a-b$ " in the B-H curve), a sharp pulse voltage indicated by $+V_{s}$ in Fig. 1(c) is induced in a search coil wound around the wire. The amplitude and the half-width of the voltage are typically $2 \mathrm{mV}$ per one turn of the search coil and 10 to $20 \mu \mathrm{s}$, respectively. These values are essentially independent of the frequency of $H_{e x t}$. This is because the parallel magnetization process is due to the Barkhausen jump and that its switching speed is constant. In order to repeat generating the pulse voltage of $+V_{s}$, the magnetization of the soft layer should be switched back. During this antiparallel magnetization process by applying $H_{b}$, a small voltage indicated $-V_{s}$ in Fig. 1(c) is also generated.

In order to generate a higher voltage, bundles of the wires were prepared. The length of the wires was $50 \mathrm{~mm}$. The asymmetric alternating magnetic fields consisted of external field $\left(H_{e x t}=80 \mathrm{Oe}\right)$ and biasing field $\left(H_{b}=20 \mathrm{Oe}\right)$ with various frequency from 0.1 to $50 \mathrm{~Hz}$ were applied. A search coil of 100 or 450 turn was used.

\section{RESULTS AND DISCUSSION}

Fig. 2 shows the peak voltage of the output $+V_{s}$ obtained from bundled wires for $n=1,2,3,5$ and 10 plotted as a function of the frequency of the applied magnetic field, where $\mathrm{n}$ is the number of bundled wires. The pickup coil of 100 turn was used. The output voltage from the bundled wires of $n<5$ is almost constant in all the measured frequency range. The output for $n=5$ and 10 at the lower frequency is decreased because 


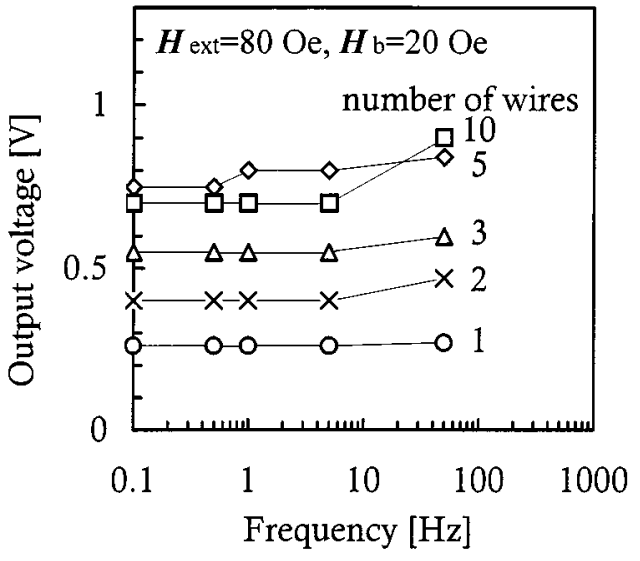

Fig. 2. Peak voltage of output generated from bundled wires of $50 \mathrm{~mm}$ as a function of frequency of applied external magnetic field $\left(H_{e x t}=80 \mathrm{Oe}\right.$ and $\left.H_{b}=20 \mathrm{Oe}\right)$.

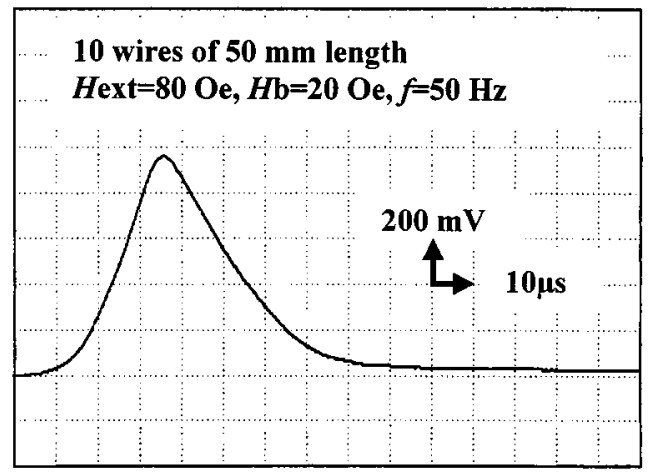

Fig. 3. Waveform of output voltage generated from bundled 10 wires of $50 \mathrm{~mm}$. The frequency of applied external magnetic field of $H_{\text {ext }}=80 \mathrm{Oe}$ and $H_{b}=20 \mathrm{Oe}$ was $50 \mathrm{~Hz}$.

of the demagnetizing effect arising from the thicker shape of the bundled wires. With increasing the number of wires, a larger demagnetizing field reduces a volume of soft layers whose magnetization is switched by the applied external magnetic field. This decrease in output is partially compensated at higher exciting frequencies, which is presumably related to the higher induced voltage of $-d \Phi / d t$. A single-peaked output waveform was obtained for all the measurements indicated in Fig. 2.

Fig. 3 shows the output waveform recorded with the bundled wires of $n=10$ at the applied field of $50 \mathrm{~Hz}$. The half width of the waveform is $26 \mu \mathrm{s}$, which is wider than the typical value of 10 to $20 \mu$ s for a single wire. This is because the timing of magnetization switching in soft layers is slightly different for each wire. This difference in timing of magnetic switching between wires is examined. Fig. 4(a) shows the magnetization of neighboring two compound magnetic wires in bundled wires. Both wires exhibit antiparallel magnetization of soft layer and hard core, and the magnetic flux is closed in each wire. When the soft layer in one of the wires, upper wire in Fig. 4(b), is reversed parallel to the hard core, the magnetic flux of this magnetically switched wire prevents the parallel magnetization of another wire. This biasing effect is so significant that the output waveform from the bundled 25 wires consists of dispersed peaks (a)

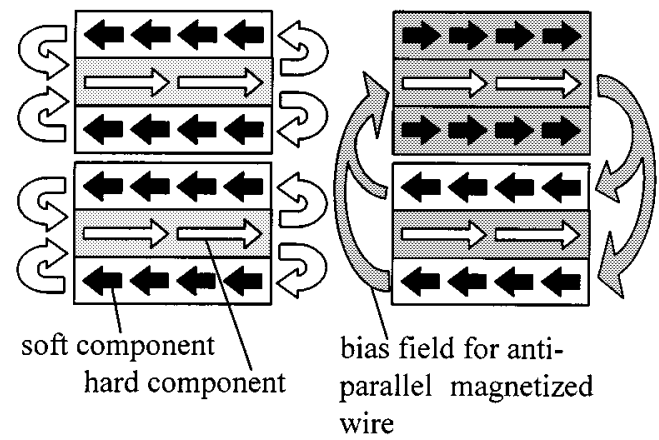

Fig. 4. Schematics of magnetization of neighboring two compound magnetic wires. Both are antiparallel magnetization (a), and one wire is parallel and another is antiparallel (b).

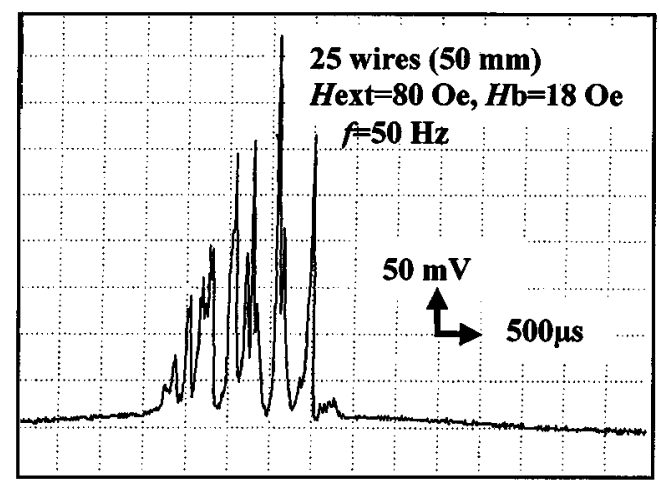

Fig. 5. Waveform of output voltage generated from bundled 25 wires of $50 \mathrm{~mm}$. The frequency of applied external magnetic field of $H_{e x t}=80 \mathrm{Oe}$ and $H_{b}=18 \mathrm{Oe}$ was $50 \mathrm{~Hz}$.

as shown in Fig. 5. As far as we investigated, the output voltage obtained from the bundled wires for $n>10$ never exhibited a single-peaked waveform for any exciting frequency of the magnetic field. But, this effect is advantageous in order to obtain a rectangular waveform (wider pulse width of the output voltage). Actually, we have obtained a high voltage of $4 \mathrm{~V}$ with a rectangle waveform for $1.2 \mathrm{~ms}$ from the bundle of 150 wires of $50 \mathrm{~mm}$ length by a 2000 turn pickup coil [2].

These results demonstrate that the compound magnetic wires can be used not only for a pulse generating device, but also a power generating device which operates at any exciting frequency. As a relatively high voltage is obtained, a power supply for electric circuits, magnetic sensor system without battery, and other applications can be developed using this device.

\section{CONCLUSION}

The output voltage generated from bundles of twisted Vicalloy wires was studied. The output did not depend on the frequency of the applied magnetic field for $n<5$. The decrease in output voltage and its frequency dependence for $n=10$ are attributed to the demagnetizing field arising from the thicker shape of the bundle. The bundled 25 wires exhibited the dispersed peaks in output waveform, which was advantageous for obtaining a rectangular waveform. A self-biasing effect is proposed in order to explain this dispersed output peaks. 


\section{ACKNOWLEDGMENT}

The authors acknowledge Dr. S. Abe at Kanagawa University, Japan for valuable discussions and the research division at Nihon System Research Institute Inc., Japan for support on the experiments.

\section{REFERENCES}

[1] S. Abe, A. Matsushita, and M. Naoe, "Dependence of large Barkhausen jump on length of a vicalloy fine wire with torsion stress," IEEE Trans. Magn., vol. 34, pp. 1318-1320, 1998.

[2] A. Matsushita and Y. Takemura, "Power generating device using compound magnetic wires," J. Appl. Phys., vol. 87, pp. 6307-6309, 2000.

[3] J. R. Wiegand and M. Velinsky, U.S. Patent \#3 820 090, 1974. 